\title{
Duration of rheumatoid arthritis and the risk of developing interstitial lung disease
}

\section{To the Editor:}

Rheumatoid arthritis is an autoimmune disease that classically presents as a symmetrical inflammatory polyarthritis. Extra-articular manifestations are prevalent, with the lungs being the most common site [1], and interstitial lung disease (ILD) being the most severe form of pulmonary involvement. In some studies, the median survival of patients with rheumatoid arthritis and a usual interstitial pneumonia (UIP) pattern on high-resolution computed tomography (HRCT) is around 3 years (similar to that in idiopathic pulmonary fibrosis (IPF) $[2,3]$ ); however, in other studies, median survival is $>7$ years $[4,5]$. When present, ILD accounts for 10-20\% of all deaths in rheumatoid arthritis [6].

There have been many reported risk factors for ILD in patients with rheumatoid arthritis, including male sex, the severity of rheumatoid arthritis and cigarette smoking [7-9]. High titres of circulating autoantibodies (rheumatoid factor, anti-cyclic citrullinated (CCP) antibodies) may also predict ILD [10, 11]. Rheumatoid arthritis-interstitial lung disease (RA-ILD) onset has previously been shown to occur in the 6th or 7th decade [12], prompting many to think of ILD as a late-occurring phenomenon in rheumatoid arthritis. In a single-centre, retrospective study of 582 subjects with rheumatoid arthritis, of which only 46 had RA-ILD, every decade increase in age at the time of rheumatoid arthritis diagnosis was associated with a $41 \%$ increased risk of RA-ILD [9]. Though it is occasionally reported in the literature that a longer duration of rheumatoid arthritis is a risk factor for ILD, there are no supporting data [13-15].

The aim of this retrospective, single-centre study, was to evaluate duration of rheumatoid arthritis as a risk factor for ILD and to determine whether duration of rheumatoid arthritis and age correlated with a radiological pattern of nonspecific interstitial pneumonia (NSIP) or UIP. Our institutional research database was queried for all subjects with a diagnosis of RA-ILD, with radiological UIP or NSIP pattern, between 2000 and $2014(\mathrm{n}=137)$. Subjects without sufficient data to determine a date of ILD diagnosis were excluded, with the final cohort including 121 subjects. The date of ILD diagnosis was defined as the earlier between the first report of ILD on imaging, the first date an outside pulmonologist confirmed a diagnosis of ILD or the first visit with an ILD specialist. A "concomitant" diagnosis of rheumatoid arthritis and ILD is defined as both conditions being diagnosed within a year of each other to account for the time from symptom onset to diagnosis. For initial analysis, all 121 patients in the cohort were included. In assessment of ILD diagnosis concomitant to, or after rheumatoid arthritis diagnosis, patients with preceding ILD diagnoses were excluded. Each subject's HRCT scan was independently reviewed by two expert thoracic radiologists ( $\mathrm{JC}$ and $\mathrm{SH}$ ) with all disagreements resolved through discussions until consensus was reached. Scans were classified as showing a pattern of UIP (RA-UIP, including both "UIP" and "probable UIP") or NSIP (RA-NSIP). Pulmonary function tests performed within 6 months of ILD diagnosis were recorded. This cohort has been previously reported on [4].

Statistical analysis was performed use SAS statistical software, Version 9.3 (SAS, Inc.; Cary, NC, USA). For initial analysis, all 121 patients in the cohort were included. In certain analyses, we excluded subjects diagnosed with ILD $>1$ year prior to the diagnosis of rheumatoid arthritis. We assessed time-to-ILD using the product-limit method, displayed results using Kaplan-Meier curves and compared curves using the log

@ERSpublications

Age of ILD onset is similar in patients with RA-UIP and RA-NSIP but duration of RA before ILD onset differs https://bit.ly/3lgjfDJ

Cite this article as: Mohning MP, Amigues I, Demoruelle MK, et al. Duration of rheumatoid arthritis and the risk of developing interstitial lung disease. ERJ Open Res 2021; 7: 00633-2020 [https://doi.org/10.1183/23120541.00633-2020]. 
a)

\begin{tabular}{|l|c|c|c|c|}
\hline & $\begin{array}{c}\text { Total } \\
\text { cohort }\end{array}$ & UIP & NSIP & p-value \\
\hline Subjects & 121 & 94 & 27 & \\
\hline Male sex & $50 \%$ & $51 \%$ & $44 \%$ & 0.663 \\
\hline Age at ILD diagnosis years & 62.5 & 62.8 & 61.7 & 0.682 \\
\hline Age at RA diagnosis years & 52 & 53.5 & 45.6 & $0.014^{*}$ \\
\hline Current or former smoker & $61 \%$ & $67 \%$ & $41 \%$ & $0.023^{*}$ \\
\hline CCP units & 138.48 & 138.8 & 137.3 & 0.950 \\
\hline RF IU.mL-1 & 654.55 & 772.9 & 258.1 & $0.0023 *$ \\
\hline Methotrexate use & $64 \%$ & $63 \%$ & $66 \%$ & 0.822 \\
\hline Biologic use & $66 \%$ & $64 \%$ & $74 \%$ & 0.365 \\
\hline FVC $\%$ pred & $70 \%$ & $70 \%$ & $69 \%$ & 0.864 \\
\hline$D_{\text {Lco } \% \text { pred }}$ & $49 \%$ & $50 \%$ & $47 \%$ & 0.632 \\
\hline
\end{tabular}
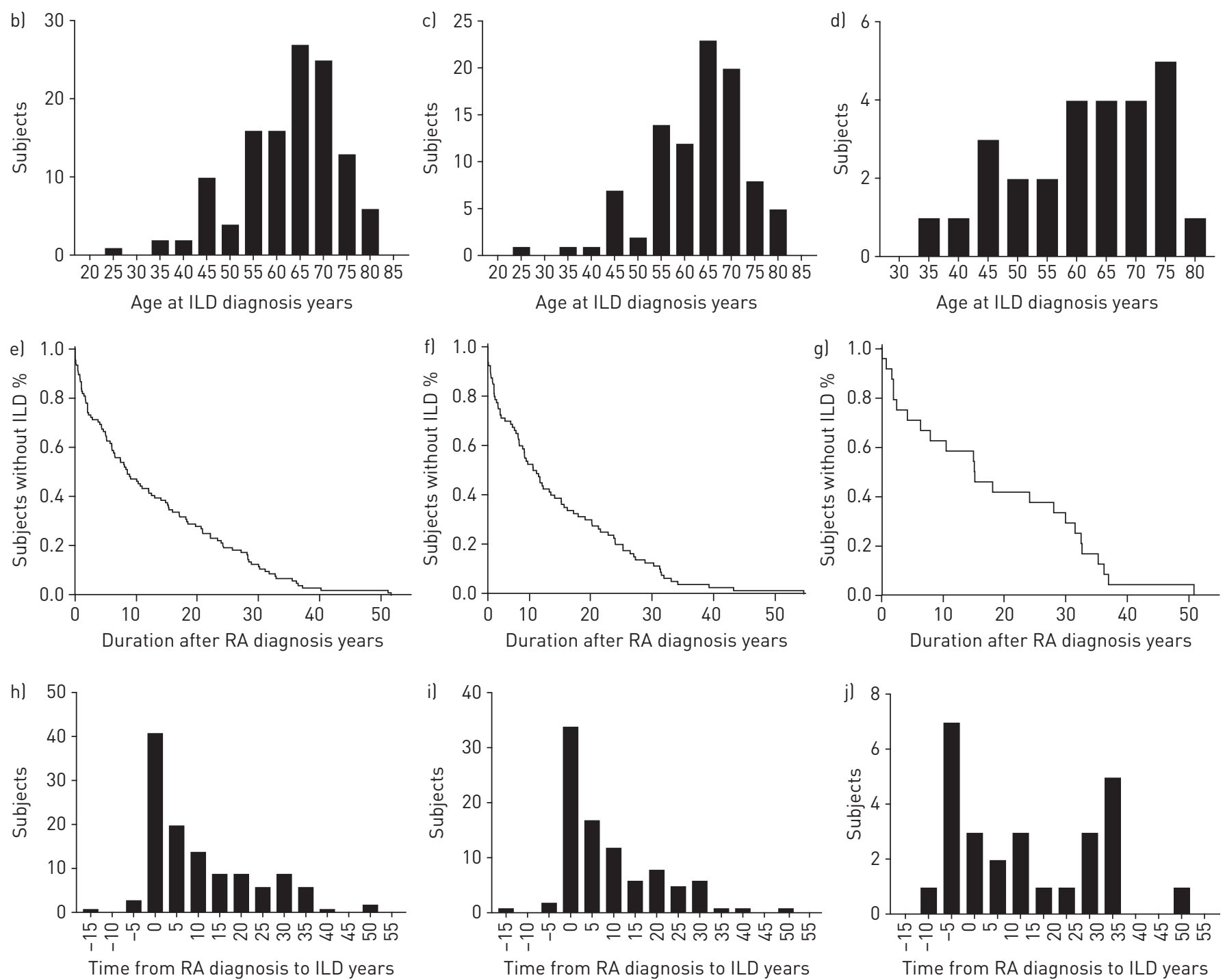

FIGURE 1 a) Baseline characteristics of cohort. Age at interstitial lung disease (ILD) diagnosis in rheumatoid arthritis (RA) patients. Histograms demonstrating number of patients at a given age (batched on 5-year intervals) at time of ILD diagnosis in b) all RA-ILD patients, and divided by high-resolution computed tomography (HRCT) radiological pattern of c) usual interstitial pneumonia (UIP) and d) nonspecific interstitial pneumonia (NSIP). Kaplan-Meier curves showing the time to development of ILD after the diagnosis of RA in e) all RA-ILD patients, and divided by HRCT radiological pattern of $f$ ) UIP and g) NSIP. Duration of RA at time of ILD diagnosis. Histograms demonstrating number of patients at a given RA duration, defined as years from diagnosis of RA (batched on 5-year intervals) at time of ILD diagnosis in h) all RA-ILD patients, and divided by HRCT radiological pattern of i) UIP and j) NSIP. CCP: cyclic citrullinated antibodies; RF: rheumatoid factor; FVC: forced vital capacity; $D_{\text {LCo }}$ : diffusing capacity of the lung for carbon monoxide. *: $p<0.05$. 
rank test. We used Cox proportional hazards to analyse time-to-ILD while controlling for potentially influential and confounding variables. We did not use selection methods but chose to include in our models clinically relevant potential confounders. We considered $\mathrm{p}<0.05$ to represent statistical significance.

As shown in figure 1a, the mean age at diagnosis of RA-ILD in our study was 63 years and did not differ between UIP and NSIP. Subjects were more likely to be male (51\%) and current or former smokers (61\%). When divided by HRCT pattern, those with UIP were more likely to have smoked and had higher rheumatoid factor titres than subjects with NSIP. Treatments were similar between the two groups. Compared to subjects with RA-NSIP, those with RA-UIP were diagnosed with rheumatoid arthritis at an older age (53.5 \pm 16 versus $45.6 \pm 14$ years, $\mathrm{p}=0.01)$. After accounting for sex and smoking, this difference remained significant $(\mathrm{p}=0.001)$.

The range in age at diagnosis of ILD was similar between RA-UIP and RA-NSIP (figure 1b-d). A significant percentage of patients had ILD either predating $(10 \%, \mathrm{n}=12)$ or concomitant with $(17 \%, \mathrm{n}=20)$ the diagnosis of rheumatoid arthritis. $49 \%$ of subjects developed ILD either before or within 5 years of the rheumatoid arthritis diagnosis and 67\% of subjects developed ILD either before or within 13 years of being diagnosed with rheumatoid arthritis.

To evaluate the duration of rheumatoid arthritis prior to the diagnosis of ILD, only those subjects whose ILD diagnosis was "concomitant" with or following the diagnosis of rheumatoid arthritis were analysed $(\mathrm{n}=109)$ (figure $1 \mathrm{e}-\mathrm{g})$. The mean duration of rheumatoid arthritis before the diagnosis of ILD was 12.4 years. Compared to NSIP, UIP was diagnosed closer to the onset of rheumatoid arthritis (10.6 versus 18.3 years from diagnosis of rheumatoid arthritis, $\mathrm{p}=0.025$, figure $1 \mathrm{~h}-\mathrm{j}$ ). When controlling for cofounders such as sex and smoking status, this difference remained significant $(\mathrm{p}=0.011)$. UIP was the ILD pattern in $84 \%$ of patients whose ILD was diagnosed prior to or concomitant with rheumatoid arthritis. When ILD was diagnosed $\geqslant 15$ years after rheumatoid arthritis, patients were more likely to have NSIP than UIP $(\mathrm{p}=0.039)$.

The development of ILD is a dreaded consequence of rheumatoid arthritis. Though there are established risk factors, there are no good prediction models to help clinicians determine who with rheumatoid arthritis will get ILD and when in the course of rheumatoid arthritis their ILD will develop. Our results add to this field of study by shedding light on the age at time of ILD diagnosis and its relationship to the time of rheumatoid arthritis diagnosis with respect to radiological ILD pattern.

Our study has limitations. This is a retrospective assessment of patients evaluated at a tertiary referral centre and may not be representative of patients seen in a community pulmonary practice. We rely on the time of clinical diagnosis of rheumatoid arthritis and ILD, and there is clear evidence that both synovitis and interstitial lung abnormalities can be present for months or years prior to a clinical diagnosis of rheumatoid arthritis or ILD. We did not analyse the impact of rheumatoid arthritis disease activity, which may have impacted the timing, subtype and severity of the ILD diagnosis.

In conclusion, we found that the pattern of ILD in patients with rheumatoid arthritis depends on the age of rheumatoid arthritis diagnosis and the duration between the diagnosis of rheumatoid arthritis and the diagnosis of ILD. ILD diagnosis clusters around the time of rheumatoid arthritis diagnosis and has a mean age of onset similar to that seen in IPF. We also found that smokers are more likely to develop a UIP pattern of lung involvement, and the majority of subjects who developed ILD prior to rheumatoid arthritis had a radiological UIP pattern. We are currently not able to predict who with rheumatoid arthritis will develop ILD, but the knowledge that ILD clusters around the time of rheumatoid arthritis diagnosis with a strong predilection for the 7th decade of life can aid clinicians in a timely diagnosis of ILD in those at risk.

Michael P. Mohning ${ }^{1}$, Isabelle Amigues ${ }^{2}$, M. Kristen Demoruelle ${ }^{2,3}$, Evans R. Fernández Pérez $\oplus^{1}$, Tristan J. Huie ${ }^{1}$, Rebecca K. Keith ${ }^{1}$, Amy L. Olson ${ }^{1}$, Zulma X. Yunt ${ }^{1}$, Jonathan H. Chung ${ }^{4}$, Stephen Hobbs ${ }^{5}$, Jeffrey J. Swigris ${ }^{1}$ and Joshua J. Solomon ${ }^{1}$

${ }^{1}$ Division of Pulmonary, Critical Care and Sleep Medicine, Dept of Medicine, National Jewish Health, Denver, CO, USA. ${ }^{2}$ Division of Rheumatology, National Jewish Health, Denver, CO, USA. ${ }^{3}$ Division of Rheumatology, University of Colorado School of Medicine, Aurora, CO, USA. ${ }^{4}$ Dept of Radiology, University of Chicago, Chicago, IL, USA. ${ }^{5}$ Dept of Radiology, University of Kentucky, Lexington, KY, USA.

Correspondence: Michael P. Mohning, National Jewish Health, 1400 Jackson St, Denver CO 80206, USA. E-mail: mohningm@njhealth.org

Received: 31 July 2020 | Accepted: 21 Sept 2020 


\section{References}

Solomon JJ, Brown KK. Rheumatoid arthritis-associated interstitial lung disease. Open Access Rheumatol 2012; 4 : 21-31.

2 Kim EJ, Elicker BM, Maldonado F, et al. Usual interstitial pneumonia in rheumatoid arthritis-associated interstitial lung disease. Eur Respir J 2010; 35: 1322-1328.

3 Solomon JJ, Ryu JH, Tazelaar HD, et al. Fibrosing interstitial pneumonia predicts survival in patients with rheumatoid arthritis-associated interstitial lung disease (RA-ILD). Respir Med 2013; 107: 1247-1252.

4 Solomon JJ, Chung JH, Cosgrove GP, et al. Predictors of mortality in rheumatoid arthritis-associated interstitial lung disease. Eur Respir J 2016; 47: 588-596.

5 Park JH, Kim DS, Park IN, et al. Prognosis of fibrotic interstitial pneumonia: idiopathic versus collagen vascular disease-related subtypes. Am J Respir Crit Care Med 2007; 175: 705-711.

6 Yunt ZX, Solomon JJ. Lung disease in rheumatoid arthritis. Rheum Dis Clin North Am 2015; 41: 225-236.

7 Demoruelle MK, Olson AL, Solomon JJ. The epidemiology of rheumatoid arthritis-associated lung disease. In Fischer A, Lee JS, eds. Lung Disease in Rheumatoid Arthritis. Cham, Springer International Publishing, 2018; pp. $45-58$.

8 Saag KG, Kolluri S, Koehnke RK, et al. Rheumatoid arthritis lung disease. Determinants of radiographic and physiologic abnormalities. Arthritis Rheum 1996; 39: 1711-1719.

9 Bongartz T, Nannini C, Medina-Velasquez YF, et al. Incidence and mortality of interstitial lung disease in rheumatoid arthritis: a population-based study. Arthritis Rheum 2010; 62: 1583-1591.

10 Alexiou I, Germenis A, Koutroumpas A, et al. Anti-cyclic citrullinated peptide-2 (CCP2) autoantibodies and extra-articular manifestations in Greek patients with rheumatoid arthritis. Clin Rheumatol 2008; 27: 511-513.

11 Mori S, Koga Y, Sugimoto M. Different risk factors between interstitial lung disease and airway disease in rheumatoid arthritis. Respir Med 2012; 106: 1591-1599.

12 Kelly CA, Saravanan V, Nisar M, et al. Rheumatoid arthritis-related interstitial lung disease: associations, prognostic factors and physiological and radiological characteristics: a large multicentre UK study. Rheumatology (Oxford) 2014; 53: 1676-1682.

13 Fong WW, Yoong JK. Interstitial lung disease and rheumatoid arthritis: a review. Proc Singapore Healthc 2015; 24 $35-41$.

14 Iqbal K, Kelly C. Treatment of rheumatoid arthritis-associated interstitial lung disease: a perspective review. Ther Adv Musculoskelet Dis 2015; 7: 247-267.

15 Georgiadis AN, Metafratzi ZM, Drosos AA. Pulmonary abnormalities in patients with early and longstanding rheumatoid arthritis. J Rheumatol 2009; 36: 444-445. 